OPEN ACCESS

Edited by:

Rosanna Cardani,

IRCCS Policlinico San Donato, Italy

Reviewed by:

Shuji Sumitomo,

The University of Tokyo, Japan

Carmelo Rodolico,

University of Messina, Italy

*Correspondence:

Yun-Fu Wang

wyfymc@sina.com;

wyfymc@163.com

Specialty section: This article was submitted to Neuromuscular Diseases, a section of the journal

Frontiers in Neurology

Received: 06 November 2019 Accepted: 12 March 2020

Published: 07 April 2020

Citation:

Wang N, Yuan J, Karim MR, Zhong P,

Sun $Y-P$, Zhang $H-Y$ and Wang $Y-F$

(2020) Effects of Mitophagy on

Regulatory T Cell Function in Patients

With Myasthenia Gravis.

Front. Neurol. 11:238.

doi: 10.3389/fneur.2020.00238

\section{Effects of Mitophagy on Regulatory T Cell Function in Patients With Myasthenia Gravis}

\author{
Na Wang ${ }^{1}$, Jiang Yuan ${ }^{1}$, Md Rezaul Karim ${ }^{1,2}$, Ping Zhong ${ }^{3}$, Yan-Peng Sun ${ }^{1}$, \\ Hong-Yan Zhang ${ }^{1}$ and Yun-Fu Wang ${ }^{1,2 *}$ \\ ${ }^{1}$ Department of Neurology, Taihe Hospital of Hubei University of Medicine, Shiyan, China, ${ }^{2}$ Biomedical Research Institute of \\ Hubei University of Medicine, Shiyan, China, ${ }^{3}$ Department of Preventive Medicine, Hubei University of Medicine, Shiyan, \\ China
}

Objective: This study was conducted to determine whether regulatory $T$ cells $\left(\mathrm{CD} 4^{+} \mathrm{CD} 25^{+} \mathrm{T}\right.$, Tregs) show abnormal mitophagy as well as the function of Tregs in patients with myasthenia gravis (MG).

Methods: $\mathrm{CD} 4^{+} \mathrm{T}$ cells and $\mathrm{CD} 4^{+} \mathrm{CD} 25^{+}$Treg cells were obtained from 15 patients with MG (MG group) and 15 controls (N group). Tregs from the MG group were subjected to rapamycin-induced culture for $48 \mathrm{~h}$ (Rapa group) and 3-methyladenine-induced culture for $48 \mathrm{~h}$ (3-MA group). The levels of mitophagy in Tregs were then observed through electron and confocal microscopy. Expression of the autophagy-related protein LC3-II was detected by western blotting, and mitochondrial function in each group was evaluated by flow cytometry. Inhibition of Treg cell proliferation was detected by flow cytometry.

Results: Mitophagy in the MG group was lower than that in the N group; it was higher in the Rapa group compared to that in the MG group and lowered in the 3-MA group than in the MG group. Expression of the autophagy-related protein LC3-II was lower in the MG group than in the N group, higher in the Rapa group than in the MG group, and lower in the 3-MA group than in the MG group. The mitochondrial membrane potential was lower in the MG group compared to that in the N group; it was higher in the Rapa group than in the MG group and lowered in the 3-MA group than in the MG group. Inhibition of Treg proliferation was lower in the MG group than in the N group; it was higher in the Rapa group than in the MG group and lowered in the 3-MA group than in the MG group.

Conclusion: The decreased mitochondrial membrane potential and mitophagy in Tregs in the MG group may be related to a decreased inhibition of Treg proliferation. The mitochondrial membrane potential was increased after adding the autophagy agent Rapa to enhance mitophagy, and the proliferation inhibition function of Tregs was also enhanced. The autophagy agent 3-MA down-regulated mitophagy, which decreased the mitochondrial membrane potential and inhibitory effect of Tregs. These results reveal the possible cellular immune mechanism of Treg dysfunction in MG.

Keywords: myasthenia gravis, mitophagy, regulatory T cell, rapamycin, 3-methyladenine 


\section{INTRODUCTION}

The acetylcholine receptor (AChR) antibody is the main mediator of cellular immune dependence and complement involvement in myasthenia gravis (MG). In AChR-related acquired autoimmune diseases, the receptor primarily involves the neuromuscular junction of the postsynaptic membrane. These diseases often manifest as a partial or full body sickness of the skeletal muscle. Muscle-specific tyrosine kinase (MuSK) receptor, low-density lipoprotein receptor-related protein 4 (LRP4) receptor, titin receptor (Titin R), and ryanodine receptor (RyR) are the few mediators in MG. Still, the clinical disease, immunopathogenesis, endplate pathology, treatments, and therapeutic targets differ $(1,2)$. In the present treatment options in MG, there are many ways to relieve symptoms and reduce the recurrence of the disease, such as cholinesterase inhibitors, glucocorticoids, immunosuppressives, anti CD20 monoclonal antibody, plasma exchange, IVIg and thymus excision therapy. All these treatment options share their unspecific mechanism, unable to cure; thus, illness aggravating, or recurrence may remain. Most of the patients need longterm therapy to maintain improvement at an effective dose of immunosuppressants $(3,4)$, or there may be side effects such as bone marrow depression, kidney damage, femoral head necrosis, etc. Some drugs are expensive and may not be affordable to many patients, such as rituxan or eculizumab $(2,5)$. Therefore, it is important to develop new methods to treat and prevent the development of MG disease by inhibiting pathological changes in the immune system.

In AChR-positive MG, the production of autoantibodies by pathogenic B cells are T cell-dependent (2). During the immune response, $\mathrm{CD} 4^{+} \mathrm{T}$ cells are activated, proliferated, and finally differentiate into Th1, Th2, Th17, and Treg cells, which mediate cellular and humoral immune responses. Th1 and Th2 cells regulate each other, Th1 and Th2 cells regulate Th17 cells, and Treg cells regulate Th17 cells, etc., so that immune effects and immunosuppression are in a delicate and complex balance. Among them, Treg cells have the functions of inhibiting the activation of autoreactive $\mathrm{T}$ cells and autoreactive $\mathrm{B}$ cells and play a major role in the regulation of the immune response and autoimmunity (6). In autoimmune diseases like $M G$, it is the imbalance between Th1 and Th2 cells and/or between Treg cells and Th17 cells (7). At present, it is believed that the abnormal number or function of $\mathrm{CD} 4{ }^{+} \mathrm{CD} 25^{+}$Treg may be the trigger point for MG (8). Therefore, in this study, we chose to start with Treg cells as the research object. Many studies have confirmed that regulatory $\mathrm{T}$ cells are involved in MG pathogenesis. The number of $\mathrm{CD}^{+} \mathrm{CD} 25^{+} \mathrm{Foxp}^{+}$Tregs and

\footnotetext{
Abbreviations: Tregs, Regulatory T cells; ROS, Reactive oxygen species; MG, Myasthenia gravis; RPMI, Roswell Park Memorial Institute; N, Normal; CFSE, Carboxyl fluorescein succinimide ester; Rapa, Rapamycin; FBS, Fetal bovine serum; 3-MA, 3-methyladenine; mTOR, Mechanistic target of rapamycin; AChR, Acetylcholine receptor; LC3-II, Microtubule-associated protein1 light chain 3; GAPDH, Glyceraldehyde-3-phosphate dehydrogenase; MuSK, Musclespecific tyrosine kinase; Titin R, Titin receptor; LRP4, low-density lipoprotein receptor-related protein 4; RyR, Ryanodine Receptor; PBMC, Peripheral blood mononuclear cell.
}

the protein expression of Foxp3 in the peripheral blood of MG patients were decreased (9). Xu et al. (10) demonstrated that the number of $\mathrm{CD} 4{ }^{+} \mathrm{CD} 25^{+} \mathrm{Foxp}^{+}$Tregs in $\mathrm{MG}$ patients was lower than that in controls. It has been reported (11) that there are lower Treg numbers and dysfunction in both experimental MG model and MG patients, it can be adjusted in a variety of ways to regulate Treg function and number, which can improve the disease condition. These reports suggest that the abnormal Treg cells are involved in MG pathogenesis.

Mitophagy has been observed as selective autophagy in various physiological processes and diseases, such as reticulocyte development (12). Geisler et al. (13) studied mitophagy and its molecular mechanism of Parkinson's disease. Mitophagy is closely related to the development, activation, and differentiation of T cells. Michalek et al. (14) found that different metabolic states determine the differentiation direction of $\mathrm{CD} 4^{+} \mathrm{T}$ cells. Treg differentiation is dependent on mitochondrial lipid oxidation, while effector $\mathrm{T}$ cells are more dependent on glycolysis. Mitophagy also plays an essential role in the development and maturation of $\mathrm{T}$ lymphocytes (15). It has been shown that in VPS34 or Atg7 knockout mice, the mitophagy of $\mathrm{CD}^{+} \mathrm{T}$ cells was decreased, while intracellular mitochondrial clearance dysfunction and reactive oxygen species (ROS) production were increased. These events lead to $\mathrm{T}$ cell dysfunction or apoptosis; thus, $\mathrm{T}$ cell metabolic states are closely related to the intracellular mitochondrial quality and quantity of stable $\mathrm{T}$ cells $(16,17)$.

The functional defects of Tregs in patients with MG may be closely related to their mitophagic abnormalities. The mitophagy function of Tregs, excess or damaged mitochondrial accumulation in cells, energy metabolism disorders, abnormal intracellular environment, activation of Tregs, proliferation disorders, and autoimmune tolerance of the body lead to the occurrence and progression of MG. To confirm this hypothesis, the relationship between mitophagy and its function in Tregs in MG were used to identify whether mitophagy occurred in Tregs in the peripheral blood of patients with MG. The mitophagy state of Tregs was regulated by 3-methyladenine (3-MA) and rapamycin (Rapa) in vitro to observe the effect of differences in mitophagy on Treg function.

\section{MATERIALS AND METHODS Specimen Collection}

In this study, all samples, including those from patients with MG and controls, were collected from the department of neurology, Taihe Hospital of Hubei University of Medicine, China. The samples were divided into two groups: (1) MG group: 15 patients (5 males and 10 females), according to the Osserman type, 9 cases of type IIA, and 6 cases of type IIB, with a mean patient age of $35.9 \pm 11.2$ years (see Table 1). (2) Control group: 15 healthy volunteers, age(s), and sex(s) were matched to the MG group, with a mean age of $36.1 \pm 9.5$ years.

Subject inclusion criteria were as follows: (1) volunteers with following medical history were excluded: acute or chronic liver disease, acute or chronic kidney disease, coronary syndrome, various infectious or other immune diseases, valvular heart disease, malignant tumor, diabetes, major 
TABLE 1 | Specimen collection of patients with MG.

\begin{tabular}{|c|c|c|c|c|c|c|c|}
\hline Serial no. & Age (years) & $\operatorname{Sex}(M / F)$ & Osserman type & Disease Duration & Antibodies & Thymus & Other autoimmune diseases \\
\hline 01 & 39 & $\mathrm{~F}$ & $\| A$ & 2 months & AChR & Thymoma (MRI) & - \\
\hline 02 & 25 & $\mathrm{~F}$ & $\| \mathrm{B}$ & 2 months & AChR & Thymic hyperplasia (CT) & $\begin{array}{l}\text { Idiopathic thrombocyto- penic } \\
\text { purpura (ITTP) }\end{array}$ \\
\hline 03 & 48 & M & $\| A$ & 10 months & AChR & Normal (CT) & - \\
\hline 04 & 23 & $\mathrm{~F}$ & $\| A$ & 2 months & $\mathrm{AChR}$ & Thymoma (CT) & Connective tissue diseases \\
\hline 05 & 45 & $\mathrm{M}$ & $\| A$ & 10 days & AChR & Thymic hyperplasia (CT) & \\
\hline 06 & 45 & $\mathrm{~F}$ & IIB & 1 year & AChR & Thymoma (CT), B2/B3 (Pathology) & - \\
\hline 07 & 47 & $\mathrm{~F}$ & $\| \mathrm{B}$ & 1 month & AChR & Normal (MRI) & $\begin{array}{l}\text { Idiopathic thrombocyto- penic } \\
\text { purpura (ITTP) }\end{array}$ \\
\hline 08 & 28 & $\mathrm{~F}$ & $\| A$ & 3 years & AChR & Normal (CT) & $\begin{array}{l}\text { Undifferentiated connective } \\
\text { tissue diseases }\end{array}$ \\
\hline 09 & 43 & M & $\| \mathrm{B}$ & 6 months & AChR & Thymoma (CT), B2 (Pathology) & - \\
\hline 10 & 41 & M & $\| A$ & 4 months & AChR & Thymoma (CT) & $\begin{array}{l}\text { Spinal cord demyelinating } \\
\text { disease }\end{array}$ \\
\hline 11 & 44 & $\mathrm{~F}$ & $\| A$ & 4 months & AChR & Thymoma (CT), B2 (Pathology) & - \\
\hline 12 & 46 & M & $\| \mathrm{B}$ & 10 days & AChR & Thymoma (CT), AB (Pathology) & - \\
\hline 13 & 40 & $\mathrm{~F}$ & $\| A$ & 2 days & AChR & Thymic hyperplasia (CT) & - \\
\hline 14 & 38 & $\mathrm{~F}$ & $\| \mathrm{B}$ & 3 years & AChR, Titin R & Normal (MRI) & - \\
\hline 15 & 45 & $\mathrm{~F}$ & $\| A$ & 1 week & AChR & Thymoma (CT), B2 /B3 (Pathology) & - \\
\hline
\end{tabular}

"-", not found with other immune-related diseases.

surgery, or severe trauma, (2) MG group: after admission, the patients were diagnosed with MG by clinical diagnosis, and all patients were Osserman type II, all the AChR-positive. These patients had not been administered anticholinergic and hormonal drugs within two weeks before the visit, and (3) Control group: healthy volunteers without the disease, was not administered anticholinergics, steroids, or other specific drugs for the past two years. In the hospital, no MG patients had been administered anticholinergics or steroid drugs before collecting a sample. Venous blood $(30 \mathrm{~mL})$ was collected with sterile anticoagulant syringes under aseptic precautions from the brachial vein. All volunteers were informed in detail before the test specific implementation process and provided consent. The hospital ethics committee approved the study.

\section{CD4 ${ }^{+}$CD25 $^{+}$Treg Separation and Drug Treatment}

Peripheral blood mononuclear cells (PBMCs) were obtained by ficoll gradient centrifugation. After removing the supernatant, the cells were counted $\left(3-4 \times 10^{7}\right.$ cells $\left./ \mathrm{mL}\right)$ using a hemocytometer. $\mathrm{CD} 4^{+} \mathrm{T}$ cells $\left(5-7 \times 10^{6}\right.$ cells $\left./ \mathrm{mL}\right)$ and $\mathrm{CD} 4^{+} \mathrm{CD} 25^{+}$Treg cells $\left(2-5 \times 10^{5}\right.$ cells $\left./ \mathrm{mL}\right)$ were obtained using an LD column, MS column, and magnetic bead sorting kit (MiltenyiBiotec, Bergisch Gladbach, Germany). CD4-FITC, CD25-PE, CD4-FITC, and CD25-PE antibodies were incubated with $\mathrm{CD} 4^{+} \mathrm{CD} 25^{+} \mathrm{T}$ cells, and the purity of $\mathrm{CD} 4^{+} \mathrm{T}$ and $\mathrm{CD} 4{ }^{+} \mathrm{CD} 25^{+} \mathrm{T}$ cells was measured by flow cytometry (BD Biosciences, Franklin Lakes, NJ, USA). The purity of $\mathrm{CD} 4^{+} \mathrm{T}$ cells was more than $95 \%$, while that of $\mathrm{CD} 4^{+} \mathrm{CD} 25^{+} \mathrm{T}$ cells was $90.2 \pm 2.6 \%$.
A $1 \mathrm{~mL}$ culture solution was prepared with $50 \mathrm{nM}$ Rapa (Sigma, St. Louis, MO, USA) and $2.5 \mathrm{mM} 3$-MA (Sigma) in $1 \mathrm{~mL}$ of culture medium (Roswell Park Memorial Institute (RPMI) $1640+10 \%$ fetal bovine serum $(\mathrm{FBS})+$ penicillin + streptomycin; Gibco, Grand Island, NY, USA) and incubated at $37^{\circ} \mathrm{C}$ for $15-30 \mathrm{~min}$. Another $2 \mathrm{~mL}$ of culture solution was prepared and incubated at $37^{\circ} \mathrm{C}$ for $15-30 \mathrm{~min}$. After obtaining $1 \mathrm{~mL}$ of culture solution, Tregs were divided into four groups: MG group1, MG group 2, MG group 3, and normal group (1 $\times 10^{5}$ cells/mL/group). For cell culture, the normal group was mixed with $1 \mathrm{~mL}$ culture solution (as described above), MG group 1 was mixed with alternative $1 \mathrm{~mL}$ culture solution, MG group 2 was mixed with $1 \mathrm{~mL}$ Rapa-containing culture solution, and MG group 3 was mixed with $1 \mathrm{~mL} 3$-MA-containing culture solution. After $48 \mathrm{~h}$ of incubation at $37^{\circ} \mathrm{C}$, the cells were collected and prepared for subsequent experiments.

In the following tests, the research team included personnel responsible for the recording of the film and data for subsequent experiments. The researchers were blinded to the sample groupings.

\section{Transmission Electron Microscopy}

$\mathrm{CD} 4{ }^{+} \mathrm{CD} 25^{+}$Tregs from each group were treated with $2.5 \%$ glutaraldehyde (diluted with $\mathrm{pH} 7.4 ; 0.1 \mathrm{mmol} / \mathrm{L}$ phosphate buffer) at $4^{\circ} \mathrm{C}$ for $2-4 \mathrm{~h}$ and then rinsed with $0.1 \mathrm{mmol} / \mathrm{L}$ phosphate buffer for $15 \mathrm{~min}$ three times. The cells were incubated in $1 \%$ osmium acid (diluted with $\mathrm{pH} 7.4 ; 0.1 \mathrm{mmol} / \mathrm{L}$ phosphate buffer) for that room temperature $\left(20^{\circ} \mathrm{C}\right)$ and then rinsed three times with $0.1 \mathrm{mmol} / \mathrm{L}$ phosphate buffer $(\mathrm{pH} 7.4)$ for $2 \mathrm{~h}$ for $15 \mathrm{~min}$ each time. Subsequently, 50, 70, 80, 90, 95, and $100 \%$ alcohol gradients were used to dehydrate the samples for $15 \mathrm{~min}$; 812 embedding agents were added to penetrate the 
cells overnight, followed by polymerization for $48 \mathrm{~h}$ at $60^{\circ} \mathrm{C}$. Next, 60-80 nM ultra-thin slices were prepared, after which uranium-lead double staining ( $2 \%$ uranium-saturated aqueous solution, lead citrate, and buffer for $15 \mathrm{~min}$ ) and drying at room temperature were performed. The total number of autophagic cells in each group (Hitachi, Tokyo, Japan) was observed with an electron microscope for five slides in each group. Then each slide was randomly chosen to calculate the total number of autophagic cells. Finally, the average number of autophagic cells in each section was analyzed statistically. The early stage of mitochondrial autophagy was distinguished based on the characteristics of mitochondria, such as bilayer membrane and cristae, and its fusion with the lysosome could be roughly recognized by a monolayer membrane or digested residue (18).

\section{Laser Confocal Microscopy}

Lysotracker and Mitotracker probe solutions ( $400 \mathrm{nM}$; catalog numbers L7528 and M7514; Life Technologies, Carlsbad, CA, USA) were incubated with the cells at $37^{\circ} \mathrm{C}$ for $30 \mathrm{~min}$. All four groups of Treg cultures were collected into four different $15 \mathrm{~mL}$ centrifuge tubes and then centrifuged, after which the supernatant was discarded. After washing with phosphate-buffered saline (PBS), $1 \mathrm{~mL}$ of pre-warm Lysotracker and Mitotracker medium was added, mixed gently, and transferred into a cell incubator. After incubation for $30 \mathrm{~min}$, the supernatants were removed by centrifugation. The samples were washed twice with PBS, and $50 \mu \mathrm{L}$ of the medium was added to resuspend the cells by thorough mixing. Next, $100 \mu \mathrm{L}$ medium was placed on a slide and covered with coverslips for immediate observation under a confocal microscope (Germa company, Germany) under the film. The fusion ratio of the two different color probes was statistically analyzed. Under a confocal laser scanning confocal microscope, the lysosomes showed red fluorescence, and mitochondria showed green fluorescence. Co-localization appeared as orange fluorescence. Under the same objective lens, the ratio of fused cell numbers to total cell numbers were calculated and statistically analyzed (i.e., ratio of orange fluorescent cells to total cells).

\section{Expression of Autophagy-Related Protein LC3-II Detected by Western Blotting}

After collecting Tregs from each group ( $n=15 /$ group), total protein was extracted, and the protein concentration was measured by the bicinchoninic acid method. Sodium dodecyl sulfate-polyacrylamide gel electrophoresis was performed for $\sim 1.5 \mathrm{~h}$. Then, proteins were transferred to a nitrocellulose membrane using a semi-dry method for $\sim 50 \mathrm{~min}$. After the transfer, $5 \%$ bovine serum albumin was incubated with the membrane at room temperature on a shaker for $30 \mathrm{~min}$ and then incubated overnight with LC3 antibody (Cell Signaling Technology, Danvers, MA, USA) and rabbit polyclonal glyceraldehyde-3-phosphate dehydrogenase (GAPDH) antibody (Hangzhou, China). Tris-buffered saline containing Tween 20 was incubated with horseradish peroxidase-labeled goat anti-rabbit IgG (Cell Signaling Technology) for $1 \mathrm{~h}$ at room temperature $\left(20-25^{\circ} \mathrm{C}\right)$; electrochemiluminescence detection was performed to evaluate the protein signal by exposure to a gel imager. Protein levels were normalized to GAPDH, and changes were determined such that the LC3-II/GAPDH ratio reflected the degree of autophagic protein expression. LC3-II is more hydrophobic than GADPH, and thus LC3-I appeared as the upper band in the electropherogram, while LC3-II was in the lower band. The ratio of LC3-II/GAPDH was calculated based on GAPDH as the internal reference level of expression for statistical analysis.

\section{Mitochondrial Membrane Potential of Tregs Detected by Flow Cytometry}

Tregs in each group ( $n=15$ /group) were collected and centrifuged. The cells were resuspended in $0.5 \mathrm{~mL}$ RPMI 1640 medium (Gibco) and mixed with $10 \mu \mathrm{g} / \mathrm{mL}$ JC-10 mitochondrial membrane potential fluorescent probe (Solarbio, Beijing, China). After incubation for $20 \mathrm{~min}$, the cells were washed twice with PBS and resuspended in PBS. Flow cytometry was performed immediately to detect the cells. JC-10 mainly aggregates to form a polymer in the mitochondrial matrix is the red fluorescence, when the membrane potential declined in the form of the monomer for the green fluorescence. The change in the green fluorescence ratio in this experiment indicated changes in the membrane potential.

\section{Treg Ability to Inhibit CD4 ${ }^{+}$T Cell Proliferation Detected by Flow Cytometry}

$\mathrm{CD}^{+} \mathrm{T}$ control group cells were isolated and resuspended in $1 \mathrm{~mL}$ of culture medium (without FBS). After adding 5 $\mu \mathrm{L} / \mathrm{mL}$ carboxyl fluoresce in succinimide ester (CFSE; Beyotime, Shanghai, China) and incubation at $37^{\circ} \mathrm{C}$ in the dark for $10 \mathrm{~min}$, $1 \mathrm{~mL}$ FBS (Gibco) was added to stop the reaction for $10 \mathrm{~min}$. The samples were washed twice with PBS and resuspended in complete medium (RPMI1640 medium + 10\% FBS + penicillin, streptomycin). CD3, CD28, and IL-2 (R\&D Systems, Minneapolis, $\mathrm{MN}$, USA), and $\mathrm{CD} 4^{+} \mathrm{CD} 25^{+}$Tregsfrom each group ( $n=15$ /group) were added to the $\mathrm{CD} 4^{+} \mathrm{CD} 25^{+} \mathrm{T}$ : $\mathrm{CD} 4^{+} \mathrm{T}$ $=1: 1$ cells for 4 days, followed by washing twice with PBS. Flow cytometry was performed, and the average fluorescence intensity of CFSE was detected using FLOWJO software (as CD $4^{+} \mathrm{T}$ cells proliferated, the average fluorescence intensity decreased, and thus the average fluorescence intensity is directly proportional to the inhibition of Treg proliferation) (19-21).

\section{Data Analysis and Statistics}

All data were expressed as the mean \pm standard deviation $(x \pm s)$. SPSS V17.0 software (SPSS, Inc., Chicago, IL, USA) was performed to analyze the data, and analysis of variance was performed to compare multiple samples. ${ }^{*} P<0.05$ was considered as statistically significant.

\section{RESULTS}

\section{Electron Microscopy}

Autophagosomes and mitochondria of $\mathrm{CD} 4{ }^{+} \mathrm{CD} 25^{+}$Tregs were observed by electron microscopy in cells from the control group, MG group, Rapa group, and 3-MA group of patients with 

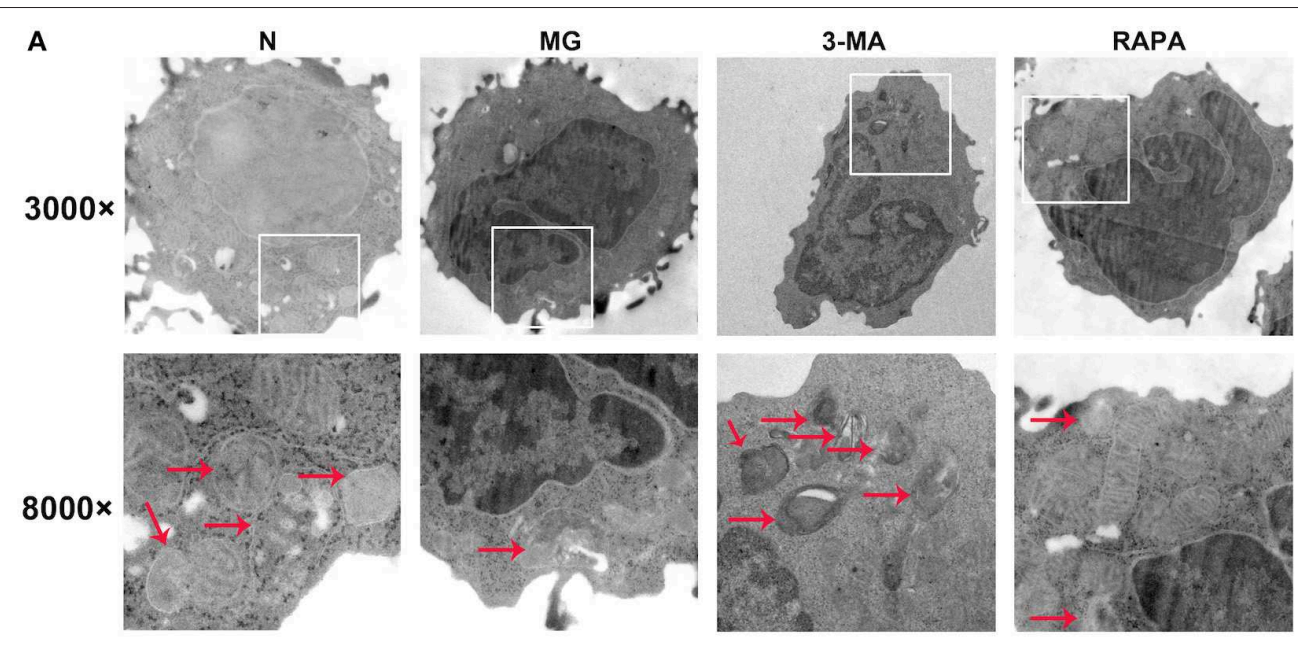

B

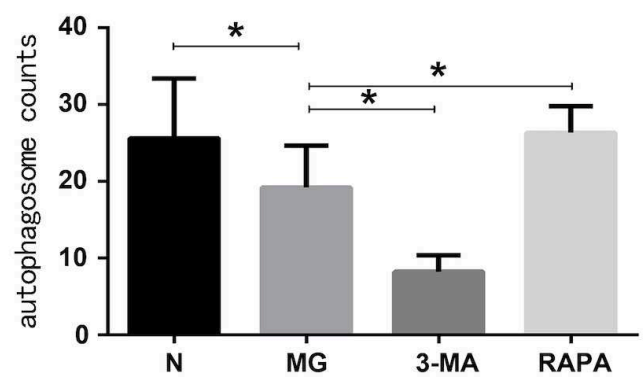

FIGURE 1 | Mitophagy under electron microscopy. (A) Mitophagy of healthy control group (N), myasthenia gravis (MG), 3-methyladenine treatment group (3-MA), and rapamycin treatment group (Rapa). In the first row is $3000 \times$, the second row is $8000 \times$. (B) N, MG, Rapa, and 3-MA four groups of statistical analyses ( ${ }^{\star} P<0.05$ ).

MG. A bilayer membrane-like structure containing cytosolic components was observed (19.20 \pm 5.49 , a sum of structures in five randomly selected cells); the cytoplasmic components were mainly mitochondrial fragments. Compared to the control group ( $25.60 \pm 7.81)$, the observed rate of autophagosome-like structures was significantly reduced $(P<0.05)$. Mitochondrial autophagy was higher in the Rapa group than in the MG group $(26.33 \pm 3.50, P<0.05)$. Mitochondrial autophagy was lower in the $3-M G$ group than in the MG group $(8.27 \pm 2.12, P<0.05$; see Figure 1).

\section{Laser Confocal Microscopy}

Each Treg group was subjected to laser confocal microscopy using Lysotracker and Mitotracker. Through laser confocal microscopy, mitochondria showed green fluorescence, lysosomes showed red fluorescence, and mitochondrion and lysosome colocalized autophagosomes showed orange (or yellow) fluorescence. The results showed that the ratio of the number of orange (or yellow) fluorescent cells to the total number of cells in the MG group $(0.321 \pm 0.085)$ was significantly lower than that of the control group $(0.603 \pm 0.133$, $P<0.05$ ). The ratio of orange (or yellow) fluorescent cells in the Rapa group was significantly increased $(0.495 \pm 0.139, P$ $<0.05)$, and the ratio in the 3 -MA group $(0.237 \pm 0.828)$ was decreased $(P<0.05)$. This suggests that patients with MG can have maturation disorders of mitochondrial autophagosomes, which were improved after Rapa treatment and were further aggravated after 3-MA treatment (see Figure 2).

\section{Autophagy Protein LC3-II Expression Levels}

Expression of the autophagy protein LC3-II was significantly decreased $(P<0.05)$ in the MG group $(0.297 \pm 0.065)$ compared to that in the normal group $(0.504 \pm 0.108)$ as detected by western blotting. The Rapa group was increased $(0.561 \pm 0.115$, $P<0.05)$, while the 3 -MA group was decreased $(0.146 \pm 0.490, P$ $<0.05)$ compared to the MG group. This suggests that autophagy in the MG group was lower than that in the control group, autophagy was increased after treatment with rapamycin but decreased after treatment with 3-MA (see Figure 3).

\section{Treg Mitochondrial Membrane Potential}

JC-10 labeling for flow cytometry analysis showed that when the mitochondrial membrane potential was high, JC-10 accumulated in the mitochondrial matrix to form a polymer, which produced red fluorescence. When the mitochondrial membrane potential was low, JC-10 did not aggregate in the mitochondrial matrix to form monomer and produced green fluorescence. The green fluorescence intensity ratio was significantly higher in the MG group $(9.28 \pm 2.09 \%)$ than in the control group $(2.73 \pm$ $0.617 \%)$ and, thus, the membrane potential was significantly decreased $(P<0.05)$. Rapa group $(3.21 \pm 1.09 \%)$ than $\mathrm{MG}$ 


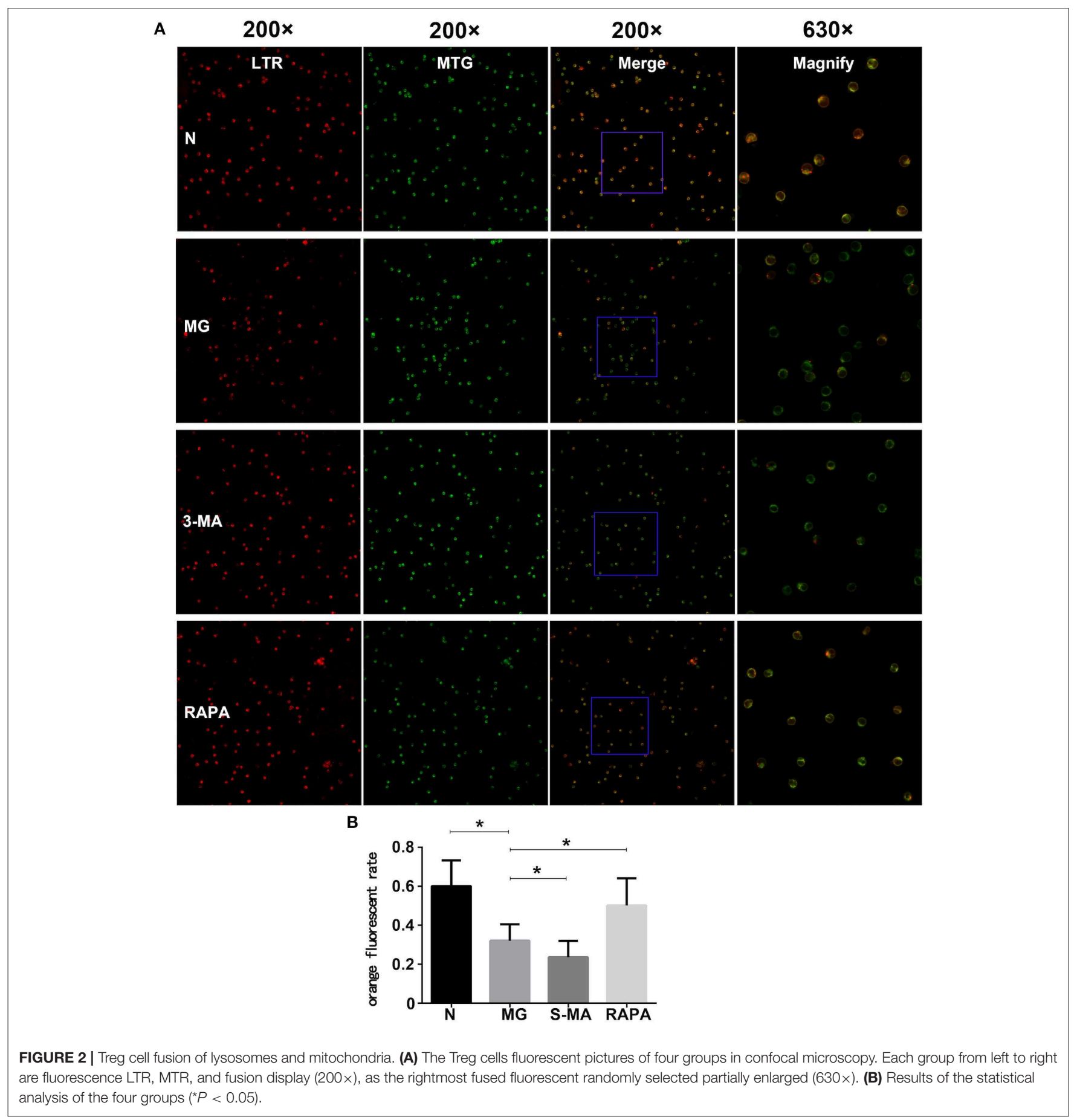

ratio decreased, membrane potential increased $(P<0.05)$, 3MA group $(11.37 \pm 3.02 \%)$ than MG ratio increased, membrane potential decreased $(P<0.05)$. These results suggest that the mitochondrial membrane potential changes with changes in the autophagy state (see Figure 4).

\section{Inhibition of Treg Proliferation}

To evaluate the inhibition of the proliferation of normal $\mathrm{CD}^{+} \mathrm{T}$ cells by Tregs, Tregs were co-cultured with CFSE-labeled normal
$\mathrm{CD} 4^{+} \mathrm{T}$ cells, and the proliferation ability of $\mathrm{CD} 4^{+} \mathrm{T}$ cells was detected by flow cytometry. CFSE released green fluorescence after covalently binding to intracellular proteins in living cells. During cell division and proliferation, fluorescence can be evenly distributed to two daughter cells, as cells proliferate, the fluorescence intensity gradually weakens, indicating a weaker ability of Tregs to inhibit proliferation. Proliferation was significantly lower in the MG group $(26.82 \pm 6.25)$ than in the normal group $(36.49 \pm 5.94, P<0.05)$; it was higher in the 


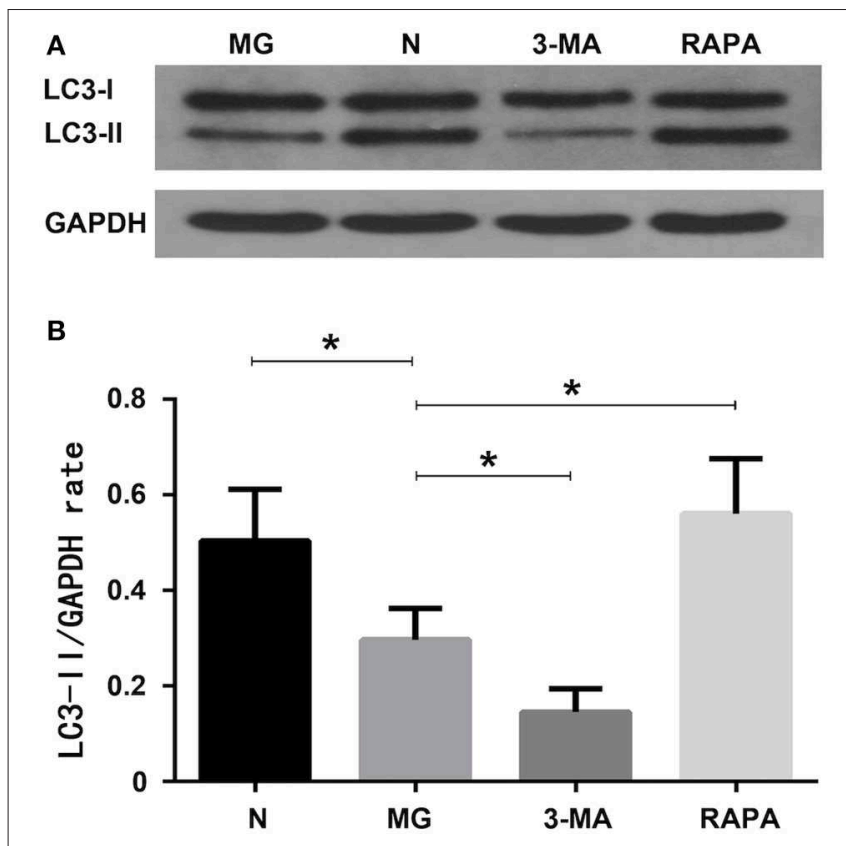

FIGURE 3 | Autophagy-related protein expression of LC3-II. (A) Western blot detection findings; (B) Statistical analysis of the four groups $\left({ }^{\star} P<0.05\right)$.

Rapa group $(40.18 \pm 4.82)$ than in the MG group $(P<0.05)$ and lower in the 3 -MA group $(20.81 \pm 6.13)$ than in the MG group $(P<0.05$; see Figure 5).

\section{DISCUSSION}

It is well-known that MG is an autoimmune disease, but the mechanisms initiation of its immune response remains unclear. There are a large number of activated autoreactive $\mathrm{CD} 4^{+} \mathrm{T}$ cells in the thymus, particularly, AChR-reactive $\mathrm{CD} 4^{+} \mathrm{T}$ cells are involved in MG. Numerous studies have shown that the number and function of Tregs are critical factors involved in the pathogenesis of MG (9-11).

In this study, peripheral blood Tregs were used to investigate the relationship between MG and mitophagy. Mitophagy is involved in various stages of $\mathrm{T}$ cell development and differentiation (22). Mitophagy is a type of intracellular antioxidative protection mechanism in activated human $\mathrm{T}$ cells, which maintains normal mitochondrial autophagy function, removes excess or damaged mitochondria in a timely manner. It also maintains normal energy metabolism and forms the basis for maintaining $\mathrm{T}$ cell homeostasis and healthy immune function $(23,24)$. Stephenson et al. (25) revealed an abnormal increase in mitochondrial volume in peripheral blood $\mathrm{T}$ cells lacking autophagy-associated protein 5 (Atg5), suggesting that autophagy plays a crucial role in mitochondrial maintenance and T cell survival. Watanabe et al. (26) found that autophagydeficient $\mathrm{CD}^{+} \mathrm{T}$ cells were more likely to induce apoptosis through the apoptotic pathway. In contrast, autophagy-deficient T-cell apoptosis occurred because of mitochondrial autophagy damage, leading to ROS accumulation and activation. Blocking autophagy in $\mathrm{T}$ cells increases the intracellular levels of mitochondria and ROS. Wei et al. (27) and Kabat et al. (28) showed that autophagy plays a critical role in regulating Tregs, suggesting that defects in autophagy decrease survival and lineage stability of peripheral blood Tregs. These studies indicate that mitophagy is essential for maintaining the number and function of Tregs. This study provides a theoretical basis for the relationship between MG and mitophagy. It has been reported that (29) peripheral blood $\mathrm{T}$ cells contain fewer mitochondria than thymic T cells; thus, mitochondrial degradation is more dependent on autophagy. Autophagy is reduced when the number of abnormal mitochondria is increased; elevated ROS in $\mathrm{T}$ cells leads to cell damage that causes disease. Abnormal mitophagy of peripheral blood Tregs may have a greater impact than thymic Treg cells on the pathogenesis of MG.

Additionally, many autoimmune diseases, such as systemic lupus erythematosus, Crohn's disease, diabetes, congenital pulmonary fibrosis, and $\mathrm{MG}$, are considered as being closely related to abnormal mitophagy (30-32). MG may, thus, be related to mitophagy. In this study, we extracted Tregs from the peripheral blood of patients with MG. We then performed electron microscopy and laser confocal microscopy (33) to qualitatively evaluate Treg mitophagy in these cells and compare to that in cells of the control group, which was significantly decreased (Figures 1, 2). LC3-II expression in the peripheral blood Tregs of patients with MG was reduced (Figure 3), indicating that the autophagy level was reduced, consistent with the qualitative test. The mitochondrial membrane potential of Tregs was detected by flow cytometry using the JC-10 probe. The mitochondrial membrane potential of Tregs in the MG group was significantly lower than that in the normal group (Figures 4A,B), destroying the mitochondrial integrity and permeability of Tregs. Intracellular mitochondrial morphological abnormalities that cannot be cleared by mitophagy result in high levels of ROS and mitochondrial toxic substances, causing the peripheral blood Tregs in MG patients to decrease or exhibit dysfunction. Finally, $\mathrm{CD}^{+} \mathrm{T}$ cell proliferation of Treg cells in patients with MG was significantly inhibited compared to that in the control group (Figures 5C,F). Previous studies (34-37) also showed a decrease in the inhibition of the proliferation of Tregs in MG patients, but the causes of abnormal cell function remained unclear, suggesting that Treg dysfunction is associated with reduced mitophagy.

3-MA and Rapa were used to regulate the autophagy of Tregs with MG. The MG group was used as a control, Rapa increased mitophagy, whereas 3-MA decreased mitophagy (Figure 1). Colocalization of LTR acidic lysosomes and MTG mitochondria was higher in the MG group and lowered in the 3-MA group (Figure 2). LC3-II expression in peripheral blood Tregs of the Rapa group was much higher than in the MG group, indicating that Rapa increases autophagy, while the 3-MA group showed lower expression of LC3-II compared to the MG group (Figure 3), indicating decreased autophagy. Finally, we detected the mitochondrial membrane potential of Tregs and inhibition of proliferation by Tregs in the Rapa and 3-MA groups. Rapa increased the mitochondrial membrane potential of Tregs (Figure 4D), altered the intracellular mitochondrial morphology, 
A

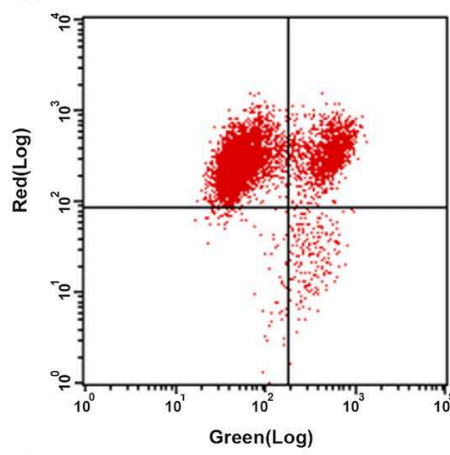

C

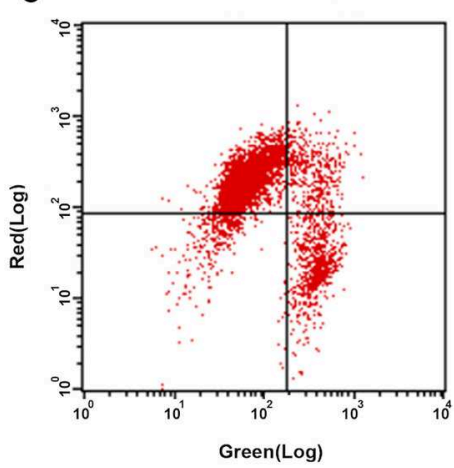

B

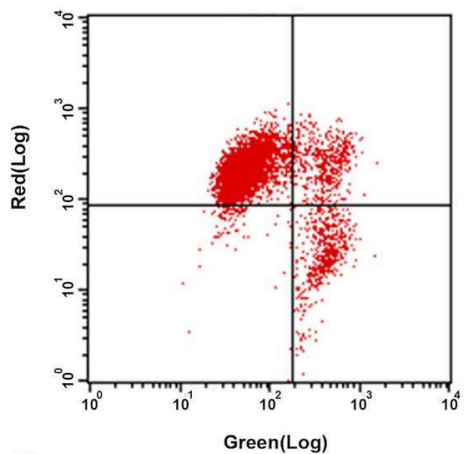

D

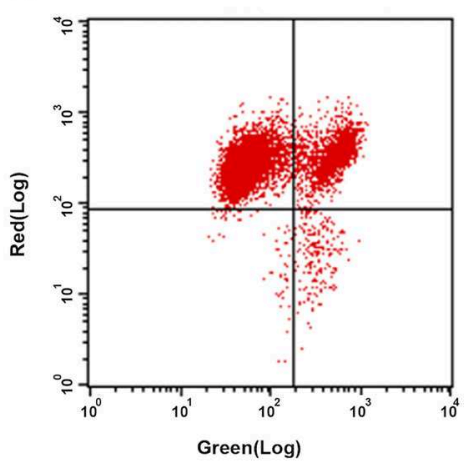

E

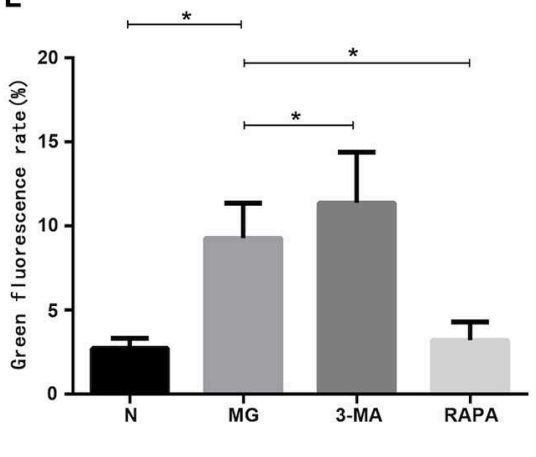

FIGURE 4 | Mitochondrial membrane potential. In the flow cytometry images, G indicates green fluorescence, R indicates red fluorescence. Mitochondrial membrane potential in the (A) healthy control group, (B) MG group, (C), 3-MA pretreatment group, (D) Rapa pretreatment group, and (E) Statistical analysis results are shown as $\left({ }^{\star} P<0.05\right)$.

increased the number and function of Tregs, and, thus, enhanced the immunoproliferative ability of Tregs (Figure 5E); 3-MA caused the opposite results (Figures 4C, 5D).

Therefore, we verified that in patients with MG, peripheral blood Tregs were increased because of reduced mitophagy. Additionally, the different mitophagy state of Tregs has a noticeable effect on the status of mitochondria and the function of Tregs. That was confirmed related to decreasing in the mitophagy level on Tregs in the peripheral blood of patients with MG.

MG is mainly treated with anti-cholinesterase drugs, immunotherapy, plasma exchange, intravenous immunoglobulin (IVIg), or thymus extraction and requires the long-term use of immunosuppressants. High-dose glucocorticoids alleviate the condition, but also cause various complications such as femoral head necrosis, osteoporosis, increased appetite, weight gain, centripetal obesity, hypertension, high blood glucose, cataracts, glaucoma, endocrine disorders, mental disorders, gastrointestinal symptoms, etc. Therefore, more effective and less reactive treatments should be identified to study the pathogenesis of MG.

Rapa (sirolimus) is an immunological preparation whose safety and effectiveness have been demonstrated. Its derivatives, such as everolimus and tacrolimus, are applied to tumors, immune diseases, and epilepsy, among other conditions. Inhibiting the mTOR pathway clearly improves the symptoms of rheumatoid arthritis, multiple sclerosis, autoimmune encephalomyelitis, and other animal models $(38,39)$. In a patient with MG complicated with iatrogenic Kaposi sarcoma, the tumor was cured, and the AChR antibody concentration was reduced after treatment with Rapa (40). Additionally, previous studies showed that Rapa effectively treated malignant thymoma (41).

Similarly, Rapa significantly improved symptoms in MG animal model rats (42). The mortality of rats after Rapa treatment was lower than that in rats administered the traditional immunosuppressant cyclophosphamide. In recent years, many studies (43-45) have shown that tacrolimus is important for treating MG, particularly for reducing the use of glucocorticoids, and is relatively safe. Our results suggest that may be Rapa intervention is a better treatment option for patients with $\mathrm{MG}$ or the other drugs, which can raise the mitophagy in Treg cell; this requires further study of larger sample size.

In this study, treatment of Tregs with Rapa increased autophagy levels, after which the function of Tregs returned to nearly normal levels, suggesting that the mTOR inhibitor Rapa can be used to treat patients with MG, but further studies are needed to determine the underlying mechanisms.

\section{CONCLUSION}

The mitophagy and function of $\mathrm{CD} 4^{+} \mathrm{CD} 25^{+}$Treg cells in patients with MG were decreased. The autophagy state of 


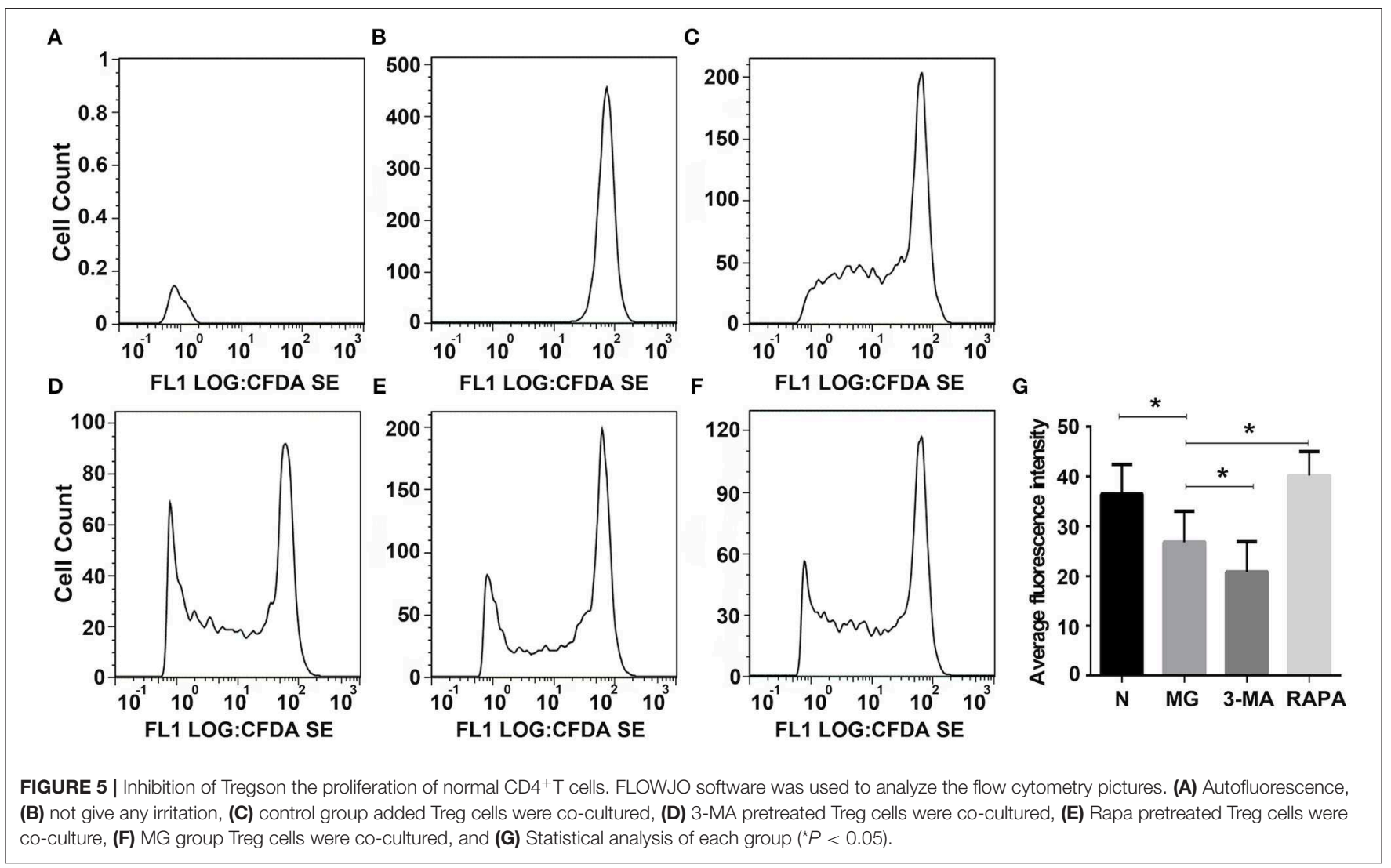

$\mathrm{CD} 4^{+} \mathrm{CD} 25^{+}$Treg cells in the peripheral blood of patients with MG was adjusted in vitro to observe changes in the immunoregulatory role of these cells. The mitophagy of $\mathrm{CD} 4{ }^{+} \mathrm{CD} 25^{+}$Treg cells may be closely related to their function, suggesting a possible mechanism for the effects of $\mathrm{CD} 4{ }^{+} \mathrm{CD} 25^{+}$Treg cells on dysfunction in patients with MG.

\section{DATA AVAILABILITY STATEMENT}

The datasets generated for this study are available on request to the corresponding author.

\section{ETHICS STATEMENT}

All subjects were informed in detail before the test specific implementation process and signed a written form of informed consent after a verbal agreement to participate in the study. The study protocols were approved by the hospital ethics committee, Taihe Hospital of Hubei University of Medicine, and institutional

\section{REFERENCES}

1. Reddel SW, Morsch M, Phillips WD. Clinical and scientific aspects of musclespecific tyrosine kinase-related myasthenia gravis. Curr Opin Neurol. (2014) 27:558-65. doi: 10.1097/WCO.0000000000000136 review board (IRB), Hubei University of Medicine, Shiyan, Hubei, China.

\section{CONSENT FOR PUBLICATION}

All the authors have agreed to publish this work.

\section{AUTHOR CONTRIBUTIONS}

All authors listed have made a substantial, direct and intellectual contribution to the work, and approved it for publication.

\section{FUNDING}

National Natural Science Foundation of China (No. 81671238).

\section{ACKNOWLEDGMENTS}

We thank the Taihe Hospital of Hubei University of Medicine for assistance throughout the research.

2. Guptill JT, Soni M, Meriggioli MN. Current treatment, emerging translational therapies, and new therapeutic targets for autoimmune myasthenia gravis. Neurotherapeutics. (2016) 13:118-31. doi: 10.1007/s13311-015-0398-y

3. Luo J, Lindstrom J, AChR-specific immunosuppressive therapy of myasthenia gravis. Biochem Pharmacol. (2015) 97:609-19. doi: 10.1016/j.bcp.2015.07.011 
4. Morren J, Li Y, Maintenance immunosuppression in myasthenia gravis, an update. J Neurol Sci. (2019) 410:116648. doi: 10.1016/j.jns.2019.116648

5. Fuchs S, Aricha R, Reuveni D, Souroujon MC. Experimental autoimmune myasthenia gravis (EAMG): from immunochemical characterization to therapeutic approaches. J Autoimmun. (2014) 54:51-9. doi: 10.1016/j.jaut.2014.06.003

6. Walker LS. Treg and CTLA-4: two intertwining pathways to immune tolerance. J Autoimmun. (2013) 45:49-57. doi: 10.1016/j.jaut.2013.06.006

7. Berrih-Aknin S. Myasthenia gravis: paradox versus paradigm in autoimmunity. J Autoimmun. (2014) 52:1-28. doi: 10.1016/j.jaut.2014.05.001

8. Pacholczyk R, Ignatowicz H, Kraj P, Ignatowicz L. Origin and T cell receptor diversity of Foxp3 + CD4+ CD25+ T cells. Immunity. (2006) 25:249-59. doi: 10.1016/j.immuni.2006.05.016

9. Zhang Y, Wang HB, Chi LJ, Wang WZ. The role of FoxP3+CD4+CD25hi Tregs in the pathogenesis of myasthenia gravis. Immunol Lett. (2009) 122:527. doi: 10.1016/j.imlet.2008.11.015

10. Xu WH, Zhang AM, Ren MS, Zhang XD, Wang F, Xu XC, et al. Changes of Treg-associated molecules on CD4+CD25 + Treg cells in myasthenia gravis and effects of immunosuppressants. J Clin Immunol. (2012) 32:975-983. doi: 10.1007/s10875-012-9685-0

11. Alahgholi-Hajibehzad M, Kasapoglu P, Jafari R, Rezaei N. The role of $\mathrm{T}$ regulatory cells in immunopathogenesis of myasthenia gravis: implications for therapeutics. Expert Rev Clin Immunol. (2015) 11:859-870. doi: 10.1586/1744666X.2015.1047345

12. Novak I, Kirkin V, McEwan DG, Zhang J, Wild P, Rozenknop A, et al. Nix is a selective autophagy receptor for mitochondrial clearance. EMBO Rep. (2010) 11:45-51. doi: 10.1038/embor.2009.256

13. Geisler S, Holmström KM, Skujat D, Fiesel FC, Rothfuss OC, Kahle PJ, et al. PINK1/Parkin-mediated mitophagy is dependent on VDAC1 and p62/SQSTM1. Nat Cell Biol. (2010) 12:119-31. doi: 10.1038/ ncb2012

14. Michalek RD, Gerriets VA, Jacobs SR, Macintyre AN, MacIver NJ, Mason EF, et al. Cutting edge: distinct glycolytic and lipid oxidative metabolic programs are essential for effector and regulatory CD4+ T cell subsets. J Immunol. (2011) 186:3299-303. doi: 10.4049/jimmunol.1003613

15. Pua HH, Guo J, Komatsu M, He YW. Autophagy is essential for mitochondrial clearance in mature T lymphocytes. J Immunol. (2009) 182:4046-55. doi: 10.4049/jimmunol.0801143

16. Willinger T, Flavell RA. Canonical autophagy dependent on the class III phosphoinositide-3 kinase Vps34 is required for naive T-cell homeostasis. Proc Natl Acad Sci USA. (2012) 109:8670-8675. doi: 10.1073/pnas.12053 05109

17. Lazarou M. Keeping the immune system in check: a role for mitophagy. Immunol Cell Biol. (2015) 93:3-10. doi: 10.1038/icb. 2014.75

18. Ding WX, Yin XM. Mitophagy: mechanisms, pathophysiological roles, and analysis. Biol Chem. (2012) 393:547-64. doi: 10.1515/hsz-2012-0119

19. Baatjes AJ, Smith SG, Watson R, Howie K, Murphy D, Larche M, et al. T regulatory cell phenotypes in peripheral blood and bronchoalveolar lavage from non-asthmatic and asthmatic subjects. Clin Exp Allergy. (2015) 45:165462. doi: 10.1111/cea.12594

20. Beurel E, Kaidanovich-Beilin O, Yeh WI, Song L, Palomo V, Michalek $\mathrm{SM}$, et al. Regulation of Th1 cells and experimental autoimmune encephalomyelitis by glycogen synthase kinase-3. J Immunol. (2013) 190:5000-11. doi: 10.4049/jimmunol.1203057

21. Shan J, Feng L, Sun G, Chen P, Zhou Y, Xia M, et al. Interplay between mTOR and STAT5 signaling modulates the balance between regulatory and effective T cells. Immunobiology. (2015) 220:510-7. doi: 10.1016/j.imbio.2014. 10.020

22. Bronietzki AW, Schuster M, Schmitz I. Autophagy in T-cell development, activation and differentiation. Immunol Cell Biol. (2015) 93:25-34. doi: 10.1038/icb.2014.81

23. Guarente L. Mitochondria-a nexus for aging, calorie restriction, and sirtuins? Cell. (2008) 132:171-6. doi: 10.1016/j.cell.2008.01.007

24. Melser S, Chatelain EH, Lavie J, Mahfouf W, Jose C, Obre E, et al. Rheb regulates mitophagy induced by mitochondrial energetic status. Cell Metab. (2013) 17:719-30. doi: 10.1016/j.cmet.2013.03.014
25. Stephenson LM, Miller BC, Ng A, Eisenberg J, Zhao Z, Cadwell K, et al. Identification of Atg5-dependent transcriptional changes and increases in mitochondrial mass in Atg5-deficient T lymphocytes. Autophagy. (2009) 5:625-635. doi: 10.4161/auto.5.5.8133

26. Watanabe R, Fujii H, Shirai T, Saito S, Ishii T, Harigae H. Autophagy plays a protective role as an anti-oxidant system in human $\mathrm{T}$ cells and represents a novel strategy for induction of T-cell apoptosis. Eur J Immunol. (2014) 44:2508-20. doi: 10.1002/eji.201344248

27. Wei J, Long L, Yang K, Guy C, Shrestha S, Chen Z, et al. Autophagy enforces functional integrity of regulatory $\mathrm{T}$ cells by coupling environmental cues and metabolic homeostasis. Nat Immunol. (2016) 17:277-85. doi: 10.1038/ni. 3365

28. Kabat AM, Harrison OJ, Riffelmacher T, Moghaddam AE, Pearson CF, Laing A, et al. The autophagy gene Atg16l1 differentially regulates Treg and TH2 cells to control intestinal inflammation. Elife. (2016) 5:e12444. doi: 10.7554/eLife.12444

29. Hubbard VM, Valdor R, Patel B, Singh R, Cuervo AM, Macian F. Macroautophagy regulates energy metabolism during effector $\mathrm{T}$ cell activation. J Immunol. (2010) 185:7349-57. doi: 10.4049/jimmunol. 1000576

30. Franke A, Balschun T, Sina C, Ellinghaus D, Häsler R, Mayr G, et al. Genomewide association study for ulcerative colitis identifies risk loci at 7q22 and 22q13 (IL17REL). Nat Genet. (2010) 42:292-4. doi: 10.1038/ng.553

31. Lee SH, Du J, Stitham J, Atteya G, Lee S, Xiang Y, et al. Inducing mitophagy in diabetic platelets protects against severe oxidative stress. EMBO Mol Med. (2016) 8:779-95. doi: 10.15252/emmm.2015 06046

32. Kobayashi K, Araya J, Minagawa S, Hara H, Saito N, Kadota T, et al. Involvement of PARK2-mediated mitophagy in idiopathic pulmonary fibrosis pathogenesis. J Immunol. (2016) 197:504-16. doi: 10.4049/jimmunol. 1600265

33. Klionsky DJ, Abdelmohsen K, Abe A, Abedin MJ, Abeliovich H, Acevedo Arozena A, et al. Guidelines for the use and interpretation of assays for monitoring autophagy (3rd edition). Autophagy. (2016) 12:1-222.

34. Rowin J, Thiruppathi M, Arhebamen E, Sheng J, Prabhakar BS, Meriggioli MN. Granulocyte macrophage colony-stimulating factor treatment of a patient in myasthenic crisis: effects on regulatory T cells. Muscle Nerve. (2012) 46:449-453. doi: 10.1002/mus.23488

35. Gertel-Lapter S, Mizrachi K, Berrih-Aknin S, Fuchs S, Souroujon MC. Impairment of regulatory $\mathrm{T}$ cells in myasthenia gravis: studies in an experimental model. Autoimmun Rev. (2013) 12:894-903. doi: 10.1016/j.autrev.2013.03.009

36. Gradolatto A, Nazzal D, Truffault F, Bismuth J, Fadel E, Foti M, et al. Both Treg cells and Tconv cells are defective in the myasthenia gravis thymus: roles of IL-17 and TNF-alpha. J Autoimmun. (2014) 52:53-63. doi: 10.1016/j.jaut.2013.12.015

37. Aricha R, Reuveni D, Fuchs S, Souroujon MC. Suppression of experimental autoimmune myasthenia gravis by autologous $\mathrm{T}$ reg $\mu$ latory cells. $J$ Autoimmun. (2016) 67:57-64. doi: 10.1016/j.jaut.2015.09.005

38. Foster JG, Blunt MD, Carter E, Ward SG. Inhibition of PI3K signaling spurs new therapeutic opportunities in inflammatory/autoimmune diseases and hematological malignancies. Pharmacol Rev. (2012) 64:1027-54. doi: $10.1124 /$ pr.110.004051

39. Thomson AW, Turnquist HR, Raimondi G. Immunoregulatory functions of m TOR inhibition. Nat Rev Immunol. (2009) 9:324-37. doi: 10.1038/nri2546

40. Krengel, S., Satzger, I., Alter, M., Kapp, A, Gutzmer, R. Remission of an iatrogenic Kaposi sarcoma in a patient with myasthenia gravis after switching immunosuppressive therapy to the mTOR inhibitor everolimus. Hautarzt. (2012) 63:573-6. doi: 10.1007/s00105-011-2274-y

41. Payne SJ, Krell J, Wilson P, Ansell W, Frampton AE, Stebbing J, et al. The efficacy of tacrolimus and sirolimus in heavily pre-treated unresectable thymic malignancies. Lung Cancer. (2013) 80:228-9. doi: 10.1016/j.lungcan.2012.12.020

42. Jing F, Yang F, Cui F, Chen Z, Ling L, Huang X, et al. Rapamycin alleviates inflammation and muscle weakness, while altering the Treg/Th17 balance in a rat model of myasthenia gravis. Biosci Rep. (2017) 37:BSR20170767. doi: 10.1042/BSR20170767 
43. Yoshikawa H, Kiuchi T, Saida T, Takamori M. Randomised, double-blind, placebo-controlled study of tacrolimus in myasthenia gravis. $J$ Neurol Neurosurg Psychiatry. (2011) 82:970-7. doi: 10.1136/jnnp-2011-300148

44. Cruz JL, Wolff ML, Vanderman AJ, Brown JN. The emerging role of tacrolimus in myasthenia gravis. Ther Adv Neurol Disord. (2015) 8:92-103. doi: $10.1177 / 1756285615571873$

45. Zhou L, Liu W, Li W, Li H, Zhang X, Shang H, et al. Tacrolimus in the treatment of myasthenia gravis in patients with an inadequate response to glucocorticoid therapy: randomized, double-blind, placebo-controlled study conducted in China. Ther Adv Neurol Disord. (2017) 10:315-25. doi: $10.1177 / 1756285617721092$
Conflict of Interest: The authors declare that the research was conducted in the absence of any commercial or financial relationships that could be construed as a potential conflict of interest.

Copyright $\odot 2020$ Wang, Yuan, Karim, Zhong, Sun, Zhang and Wang. This is an open-access article distributed under the terms of the Creative Commons Attribution License (CC BY). The use, distribution or reproduction in other forums is permitted, provided the original author(s) and the copyright owner(s) are credited and that the original publication in this journal is cited, in accordance with accepted academic practice. No use, distribution or reproduction is permitted which does not comply with these terms. 